\title{
Hospitalisation as an outcome measure
}

\section{in schizophrenia}

TOM BURNS

\begin{abstract}
Background People with
schizophrenia comprise the majority of

patients with severe mental illness

recruited to recent mental health service

studies of new teams (e.g. assertive

outreach, crisis resolution). Reduction in

hospitalisation has been the most

consistent outcome measure in these

studies, but results are inconsistent.
\end{abstract}

\begin{abstract}
Aims To understand inconsistency of results from studies using hospitalisation as an outcome measure.
\end{abstract}

\section{Method The advantages and disadvantages of hospitalisation are explored, including the ways in which it is recorded. Regional variation in outcomes and the impact of control services are reviewed.}

\section{Results Hospitalisation has face validity as an outcome but translates poorly between differing healthcare contexts. These variations can be exploited positively to distinguish potentially effective ingredients in community care (outreach, combined health and social care, team structure) from redundant components.}

Conclusions Hospitalisation is a good proxy outcome measure in schizophrenia care in randomised controlled trials, but the dangers of extrapolating to new contexts require care.

\section{Declaration of interest T.B. has} received payments for lectures and consultancies from Eli Lilly, Janssen and Otsuka in the past 5 years.
The past 25 years have witnessed an explosion in mental health services research. A 1980 review of research in community mental health services (Braun et al, 1981) cited a dozen studies and concluded that there was little evidence that the newer services sustained people longer outside hospitals. Stein \& Test's landmark study of assertive community treatment (ACT) was particularly influential because not only did it demonstrate reduced hospitalisation along with improved clinical outcomes (Stein \& Test, 1980), but the accompanying paper indicated that it could achieve this without increased costs (Weisbrod et al, 1980). Not surprisingly these two findings stimulated an enormous interest in developing and evaluating such programmes. When Mueser et al (1998) reviewed the area they were able to cite 75 good-quality studies, and when Catty et al (2002) did the same 4 years later they had over 90 studies to draw on. The vast majority of these studies focused on those with severe mental illness and invariably the samples consisted mainly of people with schizophrenia - over $80 \%$ in the UK700 trial and the Department of Veterans Affairs study (Burns et al, 1999; Rosenheck et al, 1995).

\section{HOSPITALISATION AS THE COMMON OUTCOME MEASURE}

Not surprisingly in evolving mental health services there is a considerable range of outcome measures used; these reflect both the evolution of measures and the importance placed on these various outcomes. For some researchers symptom control might have been the goal, for some social functioning and community stability, for others quality of life or risk reduction. Hospitalisation is, however, overwhelmingly the most consistent outcome reported. Hospitalisation has been assumed to be a proxy for relapse in schizophrenia in the absence of a consensus on a clinically meaningful alternative measure. The use of pre-agreed changes in symptom scores, for example a $20 \%$ decrease or reduction to an agreed level of the Positive and Negative Syndrome Scale (PANSS) score, as in trials of antipsychotics, has not found favour with clinicians for service evaluations. They are rejected because of their sensitivity to prior levels of disturbance and insensitivity to key clinical features, such as self-neglect or hostility, that have a disproportionate influence on clinical management. As a consequence, hospitalisation has come to dominate randomised controlled trials (RCTs) of community interventions because it benefits from the assumed consistency of admission threshold in any local health system despite the known variation of these between systems.

\section{Reporting of hospitalisation}

Hospitalisation is generally reported in one of three forms in community studies.

\section{Number of admissions}

This is the simplest approach and consists of recording any psychiatric admission during the study period. The frequency of admissions is usually recorded during the follow-up period and outcomes reported in terms of admitted $v$. not admitted. This reporting has the advantage that it is immediately obvious to the reader, who may know little of the local circumstances or details about admission. If there are many patients with repeated admissions during the follow-up period then the mean number of admissions in the study categories may also be presented.

\section{Time to admission}

Time to readmission has been more used in relapse prevention studies than in community care studies. The difference between the timings of relapse in the experimental and control services are presented either as mean durations or, more usually, with survival curves (e.g. Kaplan-Meier).

\section{Duration of in-patient care}

The most common presentation of hospitalisation as outcome is by days of in-patient care within the agreed follow-up period. In schizophrenia trials hospitalisation data are rarely normally distributed and usually have a pronounced skew. The majority of patients usually have no admissions and a small number of patients account for most 
of the in-patient days. Such data are best presented as medians rather than means, but planners prefer means so that they can calculate bed needs. It is increasingly common to assess bed-days with parametric statistics, presenting means, after subjecting the non-parametric results to bootstrapping techniques (Efron \& Tibshirani, 1993). An advantage of duration of care is that it permits the pooling of hospitalisation data between studies with differing follow-up periods, because the durations can be recalculated as, for example, days per month or days per year.

\section{ADVANTAGES OF HOSPITALISATION AS AN OUTCOME MEASURE}

\section{Face validity}

The most obvious advantage of hospitalisation as an outcome measure is its face validity. All clinicians have a sense of when people with schizophrenia may need admission and what admission means for the patient, the family and the service. This understanding may, of course, be more illusory than real; the threshold for admission and the experience of admission may be very different in inner-city London and in a small town in Switzerland. However, a finding that an intervention halves admission rates or duration is immediately understandable and translatable to the clinician's practice.

\section{Utility}

An understanding of changes in bed occupancy has direct utility for service planning. Indeed, it has been the translation of this outcome into projections of bed occupancy that has driven much of the research in this area and had an impact on service developments. There has been concern that the utility of research in this area has been exaggerated, either through naivety or in the service of economic imperatives. Wellrecognised factors that inflate the effectiveness of newly established services (Coid, 1994), such as charismatic leaders, the recruitment of exceptional staff and the slow accrual of complex and resistant patients, have been ignored, leading to overoptimistic bed reductions.

\section{Health economic analyses}

Because hospitalisation is such a disproportionately expensive component of mental health services - still responsible for $80 \%$ of costs in many services (Leff et al, 2000) - careful recording of it is essential to any form of cost analysis. Mental health economic analyses require careful reading and careful interpretation. More than in any other branch of medicine the extent of the costing exercise is open to real debate - how much should housing and unemployment be included, how is informal care costed, etc.? Small, apparently unconnected, changes in living conditions can completely reverse the economic benefits of interventions (McCrone et al, 1994). Where studies include hospitalisation as an outcome such complications are unlikely, but conclusions about comparative costs within services require attention to local conditions. The difference between the costs of an in-patient day and an outpatient contact with a professional are not fixed. For example, the difference between the cost of an in-patient day and a case manager contact was much greater in Stein \& Test's study (Weisbrod et al, 1980) than in the UK700 study (Byford et al, 2000). Consequently how many case manager contacts would be paid for by a saved day in hospital would be very different in the two studies.

Despite these caveats, hospitalisation data are an essential component of health economic analysis and can make a powerful case for expanding or contracting different components in an integrated service. Careful costing of hospitalisation was responsible for dispelling the early myth that deinstitutionalisation was inevitably cheaper than hospital care and helped to identify levels of disability at which hospital care was cheaper overall (Knapp et al, 1990; Hallam et al, 1994).

\section{DISADVANTAGES \\ OF HOSPITALISATION AS AN OUTCOME MEASURE}

\section{'Negative' therapeutic goal}

The most common criticism of hospitalisation as an outcome is a sense of its inadequacy in conveying normal and desirable clinical aims. Surely, its critics demand, there is more to psychiatry than simply keeping beds empty? Patient and family groups are often dismissive of a reduction in hospitalisation as evidence that services are more interested in an outcome relevant to themselves (i.e. reduced costs or administrative convenience) than to patients and families - improved well-being, quality of life and symptom control. This is a powerful criticism and not easily dismissed. Repeated attempts to contextualise hospitalisation as an outcome (explaining that it is a benchmark for clinical success, a proxy, rather than a direct measure) are necessary but often unsuccessful.

There are also disadvantages from a service development and delivery perspective of an exclusive focus on reduced hospitalisation. Sustaining mental health services relies on recruiting and retaining committed, high-quality staff, and for this the day-to-day business of care must be centred on the individual well-being of the patient directly in front of the staff member. Maintaining focus and motivation for the staff member and engaging the patient require a clear therapeutic goal that can be shared and realised in that interaction (e.g. reducing distress, improving understanding of the illness or treatment, ensuring adherence to medication). Reducing bed occupancy is not one such shared goal. Reframing this as 'promoting stability' or 'improving community tenure' goes some way to presenting it as a desirable positive goal, but statistical probabilities are weak motivators in human behaviour. Clinical experience emphasises the need to identify the clinical practices and the interpersonal and patient-centred outcomes that lead to a goal of reduced hospitalisation (Wright et al, 2004) and enshrine these in operational policies (Burns $\&$ Firn, 2002).

\section{Research distraction}

Another criticism of hospitalisation as an outcome measure is that it can distract from efforts to explore the mechanisms of schizophrenia care. This criticism certainly does have salience in service development research (Burns et al, 1999), where preoccupation with organisation has led to a relative neglect of the operative components (Wright et al, 2004), but it is probably unwarranted in the area of schizophrenia outcomes. Current research in schizophrenia care demonstrates attention to a wide range of specified interventions, both pharmacological and psychosocial, and a wide range of outcome measures.

\section{HOSPITALISATION AND RELAPSE}

\section{Independent assessment of relapse}

Hospitalisation owes its current status as a research outcome principally to its assumed equivalence with relapse. Two recent developments question this legitimacy. First, the 
increased thresholds for admission in hardpressed services or in tightly managed services may require a specific degree of severity of relapse for admission. Although agreed definitions of relapse in pharmaceutical trials have been long established based on agreed changes (either absolute or percentage point changes) in symptom ratings (such as PANSS score) they have rarely been used outside drug trials. Where there are regular ongoing assessments of clinical status as part of a study it could be possible to identify relapse independent of hospitalisation. Several ongoing naturalistic and observational studies, such as the Schizophrenia Care and Assessment Programme - UK (SCAP-UK; Burns et al, 2006), have attempted to construct relapse criteria from symptom changes, drug prescribing changes and changes in contact frequency. To date none of these attempts has been replicated in published studies.

The Lambeth Early Onset study of early intervention in psychosis has reported a reliable method for estimating relapse from regular systematised assessments of case notes (Bebbington et al, 2006). The assessed relapses were strongly correlated with independently assessed PANSS scores. Whether such an approach will erode the status of hospitalisation as an outcome measure is as yet unclear. A series of studies using such instruments might provide a guide to the relationship between relapse rates and hospitalisation rates in schizophrenia that can then be used to scale up the inevitably conservative hospitalisation rates.

\section{Crisis resolution/home treatment studies}

Unlike research into case management or assertive outreach, studies of crisis resolution/home treatment teams also use hospitalisation as a primary outcome measure but without the assumption that a change reflects a change in relapse rate. The clinical rationale of assertive outreach is that improved continuity of care leads to better clinical management and reduced relapse (Stein \& Test, 1980) and that reduced hospitalisation is a reflection of this (Marshall \& Lockwood, 1998). In studies of crisis resolution/home treatment teams, however, the intervention comprises a different style of managing relapses, not preventing them (Johnson et al, 2005; Glover et al, 2006; Killaspy et al, 2006). Thus a reduction in hospitalisation is a marker for more effective management of relapse (i.e. successful care in the home) not a marker for reduced relapse. The relationship between hospitalisation and relapse in these two different types of studies needs to be recognised for their interpretation.

\section{MISINTERPRETATION OF HOSPITALISATION AS AN OUTCOME}

Hospitalisation as an outcome measure in community studies draws its legitimacy in RCTs from the highly plausible assumption that the threshold applied in any local area will affect experimental and control groups equally. Thus any differences in hospitalisation rates can be attributed to differences between the two interventions. The dangers of extrapolating directly from model services, with their highly motivated staff, exclusion criteria and invisible incentives, have been well documented (Bachrach, 1989; Tyrer et al, 1999) although the lesson is consistently ignored. Within an individual trial, however, difference in hospitalisation is generally a reliable guide to anticipated impacts. The wider generalisability of hospitalisation is a highly complex matter and failure to give it due consideration has led to significant mistakes in policy and planning.

\section{Can hospitalisation rates be used in meta-analyses?}

Meta-analyses of medical trials consolidate the outcomes from several small trials into a single result for that outcome, treating all the data as if from a single trial. The benefits of this approach, and the worldwide Cochrane Collaboration that supports it, is that conclusions can be established earlier (thereby introducing life-saving treatments and also avoiding unnecessary subsequent trials) and with greater confidence. The delay in introducing clot-busting drugs after myocardial infarction is often cited as the most convincing case for metaanalysis (Antman et al, 1992). The importance of meta-analyses has been emphasised for mental health research because of the preponderance of small, underpowered studies (Coid, 1994). Within the Cochrane Collaboration, difference in hospitalisation rates has been the most influential outcome in meta-analyses of community mental health services (Marshall \& Lockwood, 1998; Marshall et al, 2001) although others are reported (e.g. loss to follow-up care, satisfaction with care, cost of care). Clinical and social functioning are often too inconsistently collected for influential findings to be presented.
The meta-analyses of hospitalisation for ACT teams (Marshall \& Lockwood, 1998) and case management (Marshall et al, 2001) have been consistently cited to confirm that ACT reduces the need for hospitalisation compared with standard care. As a consequence, ACT has been mandated in many US and Australian states, Canadian provinces and increasingly across Europe. In the UK ACT teams are the basis for the reorganisation of mental health services required by the NHS Plan (Department of Health, 2000), with the establishment of over 170 teams. Close examination of the forest plots indicates that there is quite a lot of heterogeneity in the results. Some caution should therefore be exercised in applying meta-analytical techniques to hospitalisation outcomes and efforts should be made to understand the source of the heterogeneity.

Two potential sources of heterogeneity are immediately clear from a cursory examination of the forest plots. First, in the ACT meta-analysis the studies demonstrating major reductions are all from the USA, and the only non-American study included (Muijen et al, 1992) demonstrates minimal reduction. In the case management analysis three of the studies are from the UK. This difference might indicate an impact of differing healthcare systems on the results of these two meta-analyses. There is also a suggestion that later studies indicate less benefit for ACT, although the difference is not as pronounced as that for the geographical differences. The importance of these observations becomes clear with the failure of any recent, high-quality European studies of ACT to replicate the reduction in hospitalisation. Indeed several recent European studies have been sufficiently powered that their failure to demonstrate reduction in hospitalisation can be interpreted as confirmation that there is no reduction. Hospitalisation is therefore not a reliable outcome in meta-analyses. Variation in hospitalisation as an outcome, on the other hand, has proved to be most useful by leading analyses that produce better understanding in service evaluations.

\section{Control services are not placebos}

Examination of the differences between US and European (predominantly UK) community care studies confirmed that the impression that US studies were more successful in reducing hospitalisation is indeed the case (Burns et al, 2002). This holds despite evidence that the interventions were 
substantially similar (Fiander et al, 2003). Home-based care in the US (the definition was widened to ensure consistency and to avoid post boc rationalisation in labelling) did reduce in-patient care by a statistically significant mean of about 10 days a year compared with standard care, whereas in European studies it increased in-patient care by a non-significant average of 3 days a year. However, the conclusion that US experimental services kept patients out of hospital more is not supported. Mean days in hospital were essentially the same for experimental service patients in the US and Europe (19 and 21 days respectively); the differences stem from the differences in hospitalisation for the control services (means of 28 and 17 days respectively).

This exploration of variation in hospitalisation data confirms our earlier call for community psychiatry studies to pay much greater attention to service characterisation and, in particular, characterisation of the control services (Burns \& Priebe, 1996). Hospitalisation as an outcome measure certainly has some generalisability, but its limitations need to be considered when it is used as a basis for service planning.

\section{Distinguishing effective ingredients}

An important consequence of the heterogeneity of hospitalisation as an outcome is that it has stimulated a search for the sources of that heterogeneity and this has helped distinguish effective from more redundant components in complex interventions. In the systematic review of home-based care by Catty et al (2002) we obtained data from the 60 of the 90 researchers to characterise their experimental services at the time of the investigations. The information was collected using 20 operationalised 'components of care', which were subjected both to cluster analysis to identify common characteristics of practice and to regression against reduction in hospitalisation to identify whether any were more strongly associated. Figure 1 shows the six regularly occurring components reported. The two found in a regression analysis to be significantly associated with reduction in hospitalisation are home visiting and joint health and social care. This is only a post hoc analysis and the sample was quite restricted. However, what it does do is indicate how hospitalisation as an outcome can be used to explore community mental health services in greater depth.
A subsequent study has demonstrated even more convincingly the utility of hospitalisation as an outcome measure to exploit differences in trials (Burns et al, 2005). Meta-regression analysis allows skewed, non-parametric data to be used in a metaanalysis (Thompson, 2001) and allows for multi-site studies to be analysed as, effectively, several independent studies. Substantially the same set of studies as those used in the home-based care review were used to obtain patient-level data. Hospitalisation data were assessed using an accepted model fidelity scale (McGrew et al, 1994). This approach demonstrated that baseline bed use was the factor most strongly associated with reduction, but of the model fidelity factors it was predominantly the structural rather than staffing characteristics of the intensive approach which accounted for the outcome differences.

\section{CONCLUSIONS}

Hospitalisation as an outcome in schizophrenia research is likely to retain an important place mainly because of its obvious utility to planners and service providers. It also has a powerful advantage in its face validity to clinicians. Its limitations are obvious - it says little about individual patient outcomes and can convey a sense of being more interested in services (in particular their costs) and the professionals that staff them than in patient welfare. A closer examination demonstrates that reducing unnecessary hospitalisation has parallelled patient and carer wishes. Survey after survey has reported the desire to remain out of hospital as much as possible (Drake \& Wallach, 1988). Given the choice, patients almost invariably opt for out-patient and community care or, failing that, day care.

Reducing unnecessary hospitalisation has also, arguably, increased the overall efficiency of mental healthcare. The disproportionate cost of in-patient care per patient contact (which is, after all, where the treatment occurs) reflects the capital costs, hotel costs and 24-hour staffing. In-patient care has declined for most physical disorders as the population increasingly has clean, well-heated accommodation affording adequate privacy. These extra costs of hospital care are justified when they add to safety or ensure adherence. However, for many patients it is not necessary and there is no clear evidence that treatments are any more effective for being delivered in hospitals than in clinics or patients' homes. Indeed, the difficulty of 'transfer of learning' from hospital to home is one of the underlying reasons for Stein \& Test's emphasis on what they call 'in vivo' care in assertive outreach (Stein \& Test, 1980).

Reducing hospitalisation is also in line with most current thinking in bioethics, where the emphasis has been on the provision of mental healthcare in the 'least restrictive' environment (Lin, 2003). Much of this ethical debate has centred around the care of legally detained patients. However, there is accumulating evidence of informal coercion in mental healthcare (Monahan et al, 2005), suggesting that the distinction between voluntary and involuntary may be better conceptualised as a gradient rather than a dichotomy (Bonnie \& Monahan, 2005). Patient and ethical views about legally enforced admission may, in some measure, also apply to most admissions.

The utility and apparent simplicity of hospitalisation as an outcome measure

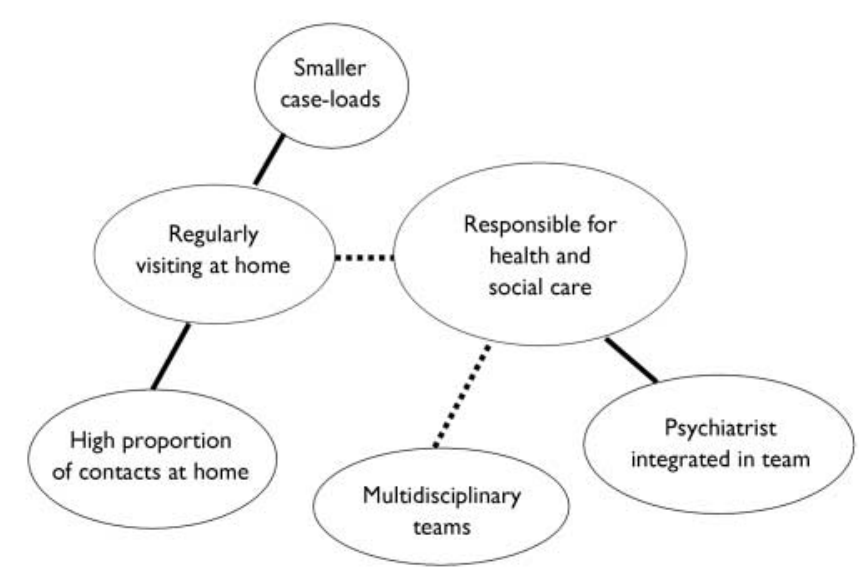

Fig. I Consistent care components of home-based care. From Wright et al (2004). Reprinted with permission. 
should not, however, blind us to its limitations. It is a good proxy for relapse in schizophrenia in well-functioning and coordinated services. However, it is a social sciences outcome that is not independent of context and it needs to be interpreted that way. Its reputation has been somewhat tarnished by overextrapolation; there is a need for greater caution in its interpretation to ensure its reputation is rehabilitated.

\section{REFERENCES}

Antman, E. M., Lau, J., Kupelnick, B., et al (1992) A comparison of results of meta-analyses of randomized control trials and recommendations of clinical experts. Treatments for myocardial infarction. JAMA, 268, 240 248.

Bachrach, L. L. (1989) The legacy of model programs. Hospital and Community Psychiatry, 40, 234-235.

Bebbington, P. E., Craig, T., Garety, P., et al (2006)

Remission and relapse in psychosis: operational

definitions based on case-note data. Psychological Medicine, 36, $155 \mid-1562$.

Bonnie, R. J. \& Monahan, J. (2005) From coercion to contract: reframing the debate on mandated community treatment for people with mental disorders. Law and Human Behavior, 29, 485-503.

Braun, P., Kochansky, G., Shapiro, R., et al (1981) Overview: deinstitutionalization of psychiatric patients, a critical review of outcome studies. American Journal of Psychiatry, I38, 736-749.

Burns, T. \& Firn, M. (2002) Assertive Outreach in Mental Health: A Manual for Practitioners. Oxford University Press.

Burns, T. \& Priebe, S. (1996) Mental health care systems and their characteristics: a proposal. Acta Psychiatrica Scandinavica, 94, 38I-385.

Burns, T., Creed, F., Fahy, T., et al (1999) Intensive versus standard case management for severe psychotic illness: a randomised trial. Lancet, 353, 2185-2189.

Burns, T., Catty, J., Watt, H., et al (2002) International differences in home treatment for mental health problems. Results of a systematic review. British Journal of Psychiatry, 18I, 375-382.

Burns, T., Marshall, M., Catty, J., et al (2005) Variable Outcomes in Case Management Trials - An Exploration of Current Theories using Meta-Regression and MetaAnalysis. Department of Health.

Burns, T., Christova, L., Cooper, S., et al (2006) Maintenance antipsychotic medication patterns in outpatient schizophrenia patients: a naturalistic cohort study. Acta Psychiatrica Scandinavica, I13, 126-134.

TOM BURNS, DSc, FRCPsych, Social Psychiatry, University Department of Psychiatry, Warneford Hospital, Headington, Oxford OX3 7JZ, UK. Email: Tom.burns@psych.ox.ac.uk

Byford, S., Fiander, M., Torgerson, D. J., et al (2000) Cost-effectiveness of intensive $v$. standard case management for severe psychotic illness. UK700 case management trial. British Journal of Psychiatry, 176, 537543.

Catty, J., Burns, T., Knapp, M., et al (2002) Home treatment for mental health problems: a systematic review. Psychological Medicine, 32, 383-401.

Coid, J. (1994) Failure in community care: psychiatry's dilemma. BMJ, 308, 805-806.

Department of Health (2000) The NHS Plan - A Plan for Investment, a Plan for Reform. Department of Health.

Drake, R. E. \& Wallach, M. A. (1988) Mental patients' attitudes toward hospitalization: a neglected aspect of hospital tenure. American Journal of Psychiatry, 145, 29-34.

Efron, B. \& Tibshirani, R. J. (1993) An Introduction to the Bootstrap. Chapman and Hall.

Fiander, M., Burns, T., McHugo, G. J., et al (2003) Assertive community treatment across the Atlantic: comparison of model fidelity in the UK and USA. British Journal of Psychiatry, 182, 248-254.

Glover, G., Arts, G. \& Babu, K. S. (2006) Crisis resolution/home treatment teams and psychiatric admission rates in England. British Journal of Psychiatry, I89, 44 I -445 .

Hallam, A., Beecham, J., Knapp, M., et al (1994) The costs of accommodation and care. Community provision for former long-stay psychiatric hospital patients. European Archives of Psychiatry and Clinical Neuroscience, 243, 304-310.

Johnson, S., Nolan, F., Pilling, S., et al (2005) Randomised controlled trial of acute mental health care by a crisis resolution team: the north Islington crisis study. BMJ, 331, 599.

Killaspy, H., Bebbington, P., Blizard, R., et al (2006) The REACT study: randomised evaluation of assertive community treatment in north London. BMJ, 332 , 815-820.

Knapp, M., Beecham, J., Anderson, J., et al (1990) The TAPS project. 3: Predicting the community costs of closing psychiatric hospitals. British Journal of Psychiatry, I57, 66I-670.

Leff, J., Trieman, N., Knapp, M., et al (2000) The TAPS Project: a report on 13 years of research, 1985-1998. Psychiatric Bulletin, 24, 165-168.

Lin, C. Y. (2003) Ethical exploration of the least restrictive alternative. Psychiatric Services, 54, 866-870.
Marshall, M. \& Lockwood, A. (1998) Assertive community treatment for people with severe mental disorders. Cochrane Library, issue 3. Update Software.

Marshall, M., Gray, A., Lockwood, A., et al (200I)

Case management for severe mental disorders. Cochrane Library, issue I. Update Software.

McCrone, P., Beecham, J. \& Knapp, M. (1994)

Community psychiatric nurse teams: cost-effectiveness of intensive support versus generic care. British Journal of Psychiatry, 165, 218-221.

McGrew, J. H., Bond, G. R., Dietzen, L., et al (1994) Measuring the fidelity of implementation of a mental health program model. Journal of Consulting and Clinical Psychology, 62, 670-678.

Monahan, J., Redlich, A. D., Swanson, J., et al (2005) Use of leverage to improve adherence to psychiatric treatment in the community. Psychiatric Services, 56 $37-44$.

Mueser, K. T., Bond, G. R., Drake, R. E., et al (1998) Models of community care for severe mental illness: a review of research on case management. Schizophrenia Bulletin, 24, 37-74.

Muijen, M., Marks, I., Connolly, J., et al (1992) Home based care and standard hospital care for patients with severe mental illness: a randomised controlled trial. BMJ, 304, 749-754.

Rosenheck, R., Neale, M., Leaf, P., et al (1995)

Multisite experimental cost study of intensive psychiatric community care. Schizophrenia Bulletin, 2I, 129-140.

Stein, L. I. \& Test, M. A. (1980) Alternative to mental hospital treatment. I. Conceptual model, treatment program, and clinical evaluation. Archives of General Psychiatry, 37, 392-397.

Thompson, S. G. (200I) Why and how sources of heterogeneity should be investigated. In Systematic Reviews in Healthcare. Meta-Analysis in Context (eds M. Egger, G. D. Smith \& D. G. Altman), pp. 157-175. BM] Books.

Tyrer, P., Coid, J., Simmonds, S., et al (1999)

Community mental health team management for those with severe mental illnesses and disordered personality. Cochrane Database Systematic of Reviews, issue 2. Update Software.

Weisbrod, B. A., Test, M. A. \& Stein, L. I. (1980) Alternative to mental hospital treatment. II. Economic benefit-cost analysis. Archives of General Psychiatry, 37, 400-405.

Wright, C., Catty, J., Watt, H., et al (2004) A systematic review of home treatment services. Classification and sustainability. Social Psychiatry and Psychiatric Epidemiology, 39, 789-796. 